\title{
Early Detection of Cancer: Evaluation of MR Imaging Grading Systems in Patients with Suspected Nasopharyngeal Carcinoma
}

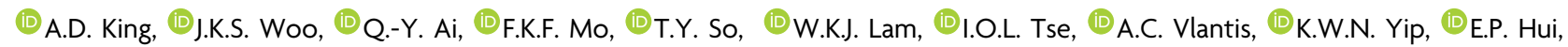

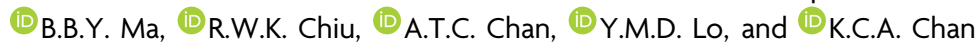

\begin{abstract}
BACKGROUND AND PURPOSE: We evaluated modifications to our contrast-enhanced MR imaging grading system for symptomatic patients with suspected nasopharyngeal carcinoma, aimed at improving discrimination of early-stage cancer and benign hyperplasia. We evaluated a second non-contrast-enhanced MR imaging grading system for asymptomatic patients from nasopharyngeal carcinoma plasma screening programs.
\end{abstract}

MATERIALS AND METHODS: Dedicated nasopharyngeal MR imaging before (plain scan system) and after intravenous contrast administration (current and modified systems) was reviewed in patients from a nasopharyngeal carcinoma-endemic region, comprising 383 patients with suspected disease without nasopharyngeal carcinoma and 383 patients with nasopharyngeal carcinoma. The modified and plain scan systems refined primary tumor criteria, added a nodal assessment, and expanded the system from 4 to 5 grades. The overall combined sensitivity and specificity of the 3 systems were compared using the extended McNemar test (a $\chi^{2}$ value $\chi_{(2)}^{2}>5.99$ indicates significance).

RESULTS: The current, modified, and plain scan MR imaging systems yielded sensitivities of $99.74 \%, 97.91 \%$, and $97.65 \%$, respectively, and specificities of $63.45 \%, 89.56 \%$ and $86.42 \%$, respectively. The modified system yielded significantly better performance than the current $\left(\chi_{(2)}^{2}=122\right)$ and plain scan $\left(\chi_{(2)}^{2}=6.1\right)$ systems. The percentages of patients with nasopharyngeal carcinoma in grades $1-2$, grade 3, and grades $4-5$ for the modified and plain scan MR imaging systems were $0.42 \%$ and $0.44 \%$; $6.31 \%$ and $6.96 \%$; and $90.36 \%$ and $87.79 \%$, respectively. No additional cancers were detected after contrast administration in cases of a plain scan graded 1-2.

CONCLUSIONS: We propose a modified MR imaging grading system that improves diagnostic performance for nasopharyngeal carcinoma detection. Contrast was not valuable for low MR imaging grades, and the plain scan shows potential for use in screening programs.

ABBREVIATION: NPC = nasopharyngeal carcinoma

T he early diagnosis of nasopharyngeal carcinoma (NPC) is essential to future improvements in patient survival and reduction of the long-term adverse effects of aggressive treatment regimens. ${ }^{1}$ Recently, we found that a high percentage of patients with early-stage NPC could be identified by population screening in Hong Kong using persistently elevated plasma Epstein-Barr virus DNA (71\% versus $20 \%$ compared with historical data). ${ }^{2}$

Received September 23, 2019; accepted after revision December 14

From the Departments of Imaging and Interventional Radiology (A.D.K., Q.Y.A., T.Y.S., K.W.N.Y.), Otorhinolaryngology, Head and Neck Surgery (J.K.S.W., A.C.V.), Clinical Oncology (F.K.F.M., E.P.H., B.B.Y.M., A.T.C.C.), and Chemical Pathology (W.K.J.L., I.O.L.T., R.W.K.C., Y.M.D.L., K.C.A.C.), and Li Ka Shing Institute of Health Sciences (F.K.F.M., W.K.J.L., I.O.L.T., E.P.H., B.B.Y.M., R.W.K.C., A.T.C.C., Y.M.D.L., K.C.A.C.), The Chinese University of Hong Kong, Prince of Wales Hospital, Hong Kong SAR, China; and State Key Laboratory of Translational Oncology (F.K.F.M., W.K.J.L., I.O.L.T., E.P.H., B.B.Y.M., R.W.K.C., A.T.C.C., Y.M.D.L., K.C.A.C.), The Chinese University of Hong Kong, Hong Kong SAR, China.

This work was supported partially by Kadoorie Charitable Foundation and the Research Grants Council of the Hong Kong Special Administrative Region, China (Project No. SEG_CUHK02, 14107216 and T12-404/11).
However, subsequent investigations must be able to detect these early cancers.

Cancers of the nasopharynx are notorious for being submucosal, located deep in the pharyngeal recess, or masked by adenoidal hyperplasia in the nasopharyngeal roof. Accordingly, endoscopic tumor detection is challenging at this site, not only for asymptomatic patients in the screening setting but also for symptomatic patients in the clinical setting. Several studies investigating patients with suspected NPC from endemic

\footnotetext{
Please address correspondence to Ann D. King, MD, Department of Imaging and Interventional Radiology, Chinese University of Hong Kong, Prince of Wales Hospital, 30-32 Ngan Shing St, Shatin, Hong Kong SAR, China; e-mail: king2015@cuhk.edu.hk

- Indicates open access to non-subscribers at www.ajnr.org

三 Indicates article with supplemental on-line table.

Indicates article with supplemental on-line photos.

http://dx.doi.org/10.3174/ajnr.A6444
}

AJNR Am J Neuroradiol 41:515-21 Mar 2020 www.ajnr.org 
Table 1: CUHK MR imaging grading systems for detection of NPC using a modified system with a contrast-enhanced scan ${ }^{\mathrm{a}}$ and a plain scan system with a noncontrast-enhanced scan $^{b}$

\begin{tabular}{|c|c|c|}
\hline MR Imaging Grade ${ }^{c}$ & Walls & Adenoid \\
\hline Grade 1: normal & $\begin{array}{l}\text { Contrast-enhanced/plain scan: thin wall, } \\
1-3 \mathrm{~mm}\end{array}$ & $\begin{array}{l}\text { Contrast-enhanced/plain scan: absent/vestigial tags/ } \\
\text { nubbin }\end{array}$ \\
\hline $\begin{array}{l}\text { Grade 2: probably benign } \\
\text { hyperplasia }\end{array}$ & $\begin{array}{l}\text { Contrast-enhanced/plain scan: diffuse } \\
\text { thickening ( }>3 \mathrm{~mm}), \text { symmetric }^{\mathrm{d}} \text { size, } \\
\text { signal intensity, and contour }\end{array}$ & $\begin{array}{l}\text { Contrast-enhanced scan: composed of Thornwaldt } \\
\text { cyst/multiple cysts, OR symmetric }{ }^{d} \text { size, signal } \\
\text { intensity, and contour with preserved symmetric } \\
\text { contrast-enhancing septa perpendicular to the roof, } \\
\text { separated by less enhancing columns (ie, stripped } \\
\text { appearance) } \\
\text { Plain scan: composed of Thornwaldt cyst/multiple cysts }\end{array}$ \\
\hline Grade 3: indeterminate & $\begin{array}{l}\text { Contrast-enhanced/plain scan: diffuse } \\
\text { thickening ( }>3 \mathrm{~mm} \text { ); asymmetric size } \\
\text { or signal intensity or contour, which is } \\
\text { nonexpansile }\end{array}$ & $\begin{array}{l}\text { Contrast-enhanced scan: asymmetric size, signal } \\
\text { intensity, OR contour with preserved or partial } \\
\text { disruption/internal distortion of contrast-enhancing } \\
\text { septa } \\
\text { Plain scan: symmetric }{ }^{d} \text { size, signal intensity, and contour }\end{array}$ \\
\hline $\begin{array}{l}\text { Grade 4: suspicious for } \\
\text { NPC }\end{array}$ & $\begin{array}{l}\text { Contrast-enhanced/plain scan: diffuse } \\
\text { thickening ( }>3 \mathrm{~mm} \text { ); asymmetric size } \\
\text { or signal intensity or contour, which is } \\
\text { expansile (superficial or deep margins) }\end{array}$ & $\begin{array}{l}\text { Contrast-enhanced scan: absent contrast-enhancing } \\
\text { septa in a focal adenoid, OR external distortion of } \\
\text { contrast-enhancing septa by an adjacent roof mass } \\
\text { Plain scan: asymmetric size, signal intensity, or contour }\end{array}$ \\
\hline Grade 5: probably NPC & & \\
\hline $5 a$ & $\begin{array}{l}\text { Contrast-enhanced/plain scan: focal } \\
\text { mass }\end{array}$ & $\begin{array}{l}\text { Contrast-enhanced scan: absent contrast-enhancing } \\
\text { septa in an adenoid filling the whole roof on at least } 1 \\
\text { section } \\
\text { Plain scan: no grade }\end{array}$ \\
\hline $5 b$ & \multirow{2}{*}{\multicolumn{2}{|c|}{$\begin{array}{l}\text { Contrast-enhanced/plain scan: spread outside the nasopharynx (superficial or deep) } \\
\text { Contrast-enhanced/plain scan: metastatic retropharyngeal or upper cervical nodes }\end{array}$}} \\
\hline $5 c$ & & \\
\hline
\end{tabular}

Note: - CUHK indicates Chinese University of Hong Kong; NPC, nasopharyngeal carcinoma.

${ }^{a}$ Modified MR imaging protocol: T2- and T-weighted images without and with intravenous contrast enhancement.

b Plain scan MR imaging protocol: T2- and T1-weighted images without intravenous contrast enhancement.

${ }^{C}$ For the modified grading system, the grade for contrast-enhanced images outranks the grade for non-contrast-enhanced images.

${ }^{\mathrm{d}}$ Symmetry refers to a comparison of the right and left halves of the nasopharynx for size, signal intensity, and contour; cysts do not contribute to wall or adenoid asymmetry or to distortion of adenoidal septa.

e Diagnosis of a metastatic node is based on size (minimum axial nodal diameter: retropharyngeal, $\geq 6$ mm; jugulodigastric, $\geq 11 \mathrm{~mm}$; all other nodes, $\geq 10$ mm or groups of $\geq 3$ nodes with a minimal axial diameter of $\geq 8 \mathrm{~mm}$ ) or any node with necrosis or extracapsular spread.

regions, ${ }^{3,4}$ as well as nonendemic regions, ${ }^{5}$ have shown that MR imaging can detect NPCs hidden from endoscopic view. In an MR imaging study of predominantly symptomatic patients referred to the outpatient clinic with suspected NPC, we found that MR imaging detects the $10 \%$ of tumors that are hidden from endoscopic view. ${ }^{3}$ This figure rises to $17 \%$ in asymptomatic patients screened for NPC using persistently elevated plasma Epstein-Barr virus DNA. ${ }^{4}$ In both studies, MR imaging detected tumors up to 3 years before they became visible endoscopically. ${ }^{4,6} \mathrm{MR}$ imaging, therefore, has a complementary role in early NPC detection.

We have shown that the standardized MR imaging grading system used at our institution has a high sensitivity for NPC detection, ${ }^{3,4,7}$ which includes early-stage tumors hidden on the endoscopic examination that tend to form a focal mass in the roof or pharyngeal recess. ${ }^{3,4}$ However, some early-stage cancers confined within the nasopharynx are diffuse rather than focal. In these diffuse early-stage NPCs, the wall thickening is usually greater on one side of the nasopharynx, so discrimination of NPC from benign hyperplasia of the walls and adenoid relies heavily on finding asymmetry on bilateral nasopharyngeal MR imaging evaluations. ${ }^{3,4,8,9}$ Unfortunately, on scrutiny of the images for small early-stage tumors, benign hyperplasia is often slightly asymmetric. This characteristic is challenging in screened subjects with benign lesions and could produce false-positive findings leading to unnecessary alarm and resource usage. ${ }^{4}$ Moreover, in a study of a large group of stage T1 NPCs confined to the nasopharynx, we observed that a small number of these early-stage tumors were symmetrically diffuse and overlapped in appearance with benign hyperplasia. ${ }^{8}$ These early-stage tumors could potentially reduce the high sensitivity of MR imaging.

In the first part of the study, we modified the current contrast-enhanced MR imaging grading system, which can be used worldwide as a complementary investigation in symptomatic patients with suspected NPC when endoscopic examination findings are negative or indeterminate. The aim was to improve the specificity of MR imaging and maintain a high sensitivity, by adding focal expansion in asymmetric diffuse thickening, absent or external distortion of contrast-enhancing adenoidal septa, and the presence of metastatic nodes to indicate NPC. In the second part of the study, we evaluated a new plain scan MR imaging grading system involving a short protocol without intravenous contrast for use in screening programs in endemic regions such as east and southeast parts of Asia, where the incidence in middle-aged men is up to 35 per $100,000 .^{10}$ Most patients with persistently elevated plasma or serology markers for NPC referred for investigation do not have NPC. In these patients, it would be advantageous to limit the use of gadolinium MR imaging contrast 
Table 2: Patients with and without NPC in each MR imaging grade as determined using the current, modified, and plain scan MR imaging grading systems

\begin{tabular}{|c|c|c|c|c|}
\hline \multirow[b]{2}{*}{ Grading Systems } & \multicolumn{2}{|c|}{ No. of Patients without NPC } & \multicolumn{2}{|c|}{ No. of Patients with NPC } \\
\hline & Nodes Not Assessed & Nodes Assessed & Nodes Not Assessed & Nodes Assessed \\
\hline \multicolumn{5}{|l|}{ Grade 1} \\
\hline Current & 69 & - & 0 & - \\
\hline Modified & 69 & 69 & 0 & 0 \\
\hline Plain scan & 79 & 78 & 0 & 0 \\
\hline \multicolumn{5}{|l|}{ Grade 2} \\
\hline Current & 174 & - & 1 & - \\
\hline Modified & 174 & 170 & 1 & 1 \\
\hline Plain scan & 147 & 146 & 2 & 1 \\
\hline \multicolumn{5}{|l|}{ Grade 3} \\
\hline Current & 132 & - & 47 & - \\
\hline Modified & 109 & 104 & 13 & 7 \\
\hline Plain scan & 112 & 107 & 15 & 8 \\
\hline \multicolumn{5}{|l|}{ Grade 4} \\
\hline Current & 8 & - & 335 & - \\
\hline Modified & 28 & 26 & 91 & 26 \\
\hline Plain scan & 42 & 38 & 123 & 32 \\
\hline \multicolumn{5}{|l|}{ Grade 5} \\
\hline Current & - & - & - & - \\
\hline Modified & 3 & 14 & 278 & 349 \\
\hline Plain scan & 3 & 14 & 243 & 342 \\
\hline
\end{tabular}

Note:-- indicates not applicable; NPC, nasopharyngeal carcinoma.

agents. Furthermore, a short plain scan has greater potential for use in screening programs in NPC endemic regions to prioritize or even select patients for endoscopic examination. Finally, observers with different levels of experience in head and neck MR imaging tested the modified and plain scan MR imaging systems.

\section{MATERIALS AND METHODS \\ Patients}

This retrospective evaluation of MR imaging grading systems in patients who underwent MR imaging for the staging of known NPC or investigation of suspected NPC was approved by the institutional review board (the Chinese University of Hong Kong), which waived the requirement for written informed consent. Nasopharyngeal MR imaging data obtained from 766 patients before and after intravenous contrast were reviewed. Patients did not have a history of head and neck cancer before they underwent the MR imaging for suspected or biopsy-proved NPC. The first group comprised 383 patients without NPC (304 men and 79 women; mean age, 52 years; age range, $18-83$ years) who had undergone an endoscopic examination and MR imaging for suspected NPC (based on clinical symptoms, clinical signs, or elevated blood markers for NPC) between 2005 and 2016, but had not been diagnosed with head and neck cancer (minimum follow-up of 2 years). Details are shown in On-line Fig 1. These included 358 patients recruited for 2 previous prospective NPCdetection studies. ${ }^{3,4}$ The second group comprised a similar sample size of 383 patients referred for suspected NPC (based on clinical symptoms, clinical signs, or elevated blood markers for NPC) who had biopsy-proved undifferentiated NPC (282 men and 101 women; mean age, 53 years; age range, 19-92 years). These patients were randomly selected from consecutive patients with NPC scanned between 2005 and 2016 according to the expected T-stage ${ }^{11}$ distribution in a screened population: stage T1 (nasopharynx), 246 patients (64.2\%); T1 (nasal cavity/oropharynx), 13 (3.4\%); T2, 33 (8.6\%); T3, 80 (20.9\%); and T4, 11 (2.9\%). These include 134 patients with $\mathrm{T} 1$ cancer reported previously. ${ }^{8}$

\section{MR Imaging Acquisition}

Targeted nasopharyngeal MR imaging was performed using a $1.5 \mathrm{~T}$ or 3T whole-body MR imaging system (Philips Healthcare, Best, the Netherlands). During a scan duration of approximately 15-20 minutes, axial fat-suppressed T2-weighted, coronal T2weighted, and axial T1-weighted images, and axial and coronal T1-weighted images were obtained after a bolus injection of $0.1 \mathrm{mmol}$ per kilogram of body weight of gadoterate meglumine (Dotarem; Guerbet, Aulnay-sous-Bois, France).

\section{MR Imaging Grading}

The current MR imaging grading system ${ }^{3}$ is shown in the Online Table. The modified and plain scan MR imaging grading systems are shown in Table 1 (which differ only in grading the adenoid because contrast-enhancing septa cannot be assessed on the plain scan) and are illustrated in On-line Figs 2 and 3, respectively. The diagnosis of a metastatic node was based on recognized imaging criteria shown in Table $1 .{ }^{12,13}$ The MR imaging data were graded by readers blinded to the diagnosis of NPC or no NPC. Initially, non-contrast-enhanced images (T2- and T1weighted images) were graded according to the proposed plain scan MR imaging grading system. Subsequently, the full scan, including contrast-enhanced images (T2- and T1-weighted images pre- and post-contrast enhancement) was graded according to our current and proposed modified MR imaging grading systems.

Results were from the MR imaging data assessed by a radiologist with $>20$ years of experience in MR imaging of NPC (observer 1 ). The grading systems were tested by 2 further observers, a researcher with 5 years of experience in MR imaging of NPC 
Table 3: Diagnostic performance of MR imaging after grouping of grades into benign or malignant according to grades in the current, modified, and plain scan grading systems

\begin{tabular}{|c|c|c|c|c|c|c|c|c|c|}
\hline MR Imaging Grade Grouping to Indicate NPC & FP & FN & TP & $\mathrm{TN}$ & Sen \% & Spec \% & PPV \% & NPV \% & Accuracy \% \\
\hline \multicolumn{10}{|l|}{ Grades in the current system } \\
\hline Primary tumor $3,4^{a}$ & 140 & 1 & 382 & 243 & 99.74 & 63.45 & 73.18 & 99.59 & 81.59 \\
\hline Primary tumor only 4 & 8 & 48 & 335 & 375 & 87.47 & 97.91 & 97.67 & 88.65 & 92.69 \\
\hline \multicolumn{10}{|l|}{ Grades in the modified system } \\
\hline Primary tumor $3,4,5$ & 140 & 1 & 382 & 243 & 99.74 & 63.45 & 73.18 & 99.59 & 81.59 \\
\hline Primary tumor 4,5 & 31 & 14 & 369 & 352 & 96.34 & 91.91 & 92.25 & 96.17 & 94.13 \\
\hline Primary tumor $4,5+$ node $5^{a}$ & 40 & 8 & 375 & 343 & 97.91 & 89.56 & 90.36 & 97.72 & 93.73 \\
\hline \multicolumn{10}{|l|}{ Grades in the plain scan system } \\
\hline Primary tumor 4,5 & 45 & 17 & 366 & 338 & 95.56 & 88.25 & 89.05 & 95.21 & 91.91 \\
\hline Primary tumor $4,5+$ node $5^{\text {a }}$ & 52 & 9 & 374 & 331 & 97.65 & 86.42 & 87.79 & 97.35 & 92.04 \\
\hline
\end{tabular}

Note:-FP indicates false-positive; FN, false-negative; TP, true-positive; TN, true-negative; Sen, sensitivity; Spec, specificity; PPV, positive predictive value; NPV, negative predictive value.

${ }^{a}$ Combination of grades in the respective current, modified, and plain scan systems in Table 2.

(observer 2) and a general radiologist with a 1-year postqualification in radiology (after training on 50 patients not included the study analysis comprising 25 without and 25 with NPC with a distribution of T-stages similar to that in the study) (observer 3 ).

\section{Statistical Analysis}

The performance of MR imaging for NPC detection and classification of lesions as benign and malignant was assessed by calculating the sensitivity, specificity, positive predictive value, negative predictive value, and accuracy based on groupings of grades. The concordance rates between MR imaging grading systems were analyzed according to Hawass. ${ }^{14}$ The McNemar test was used to test the sensitivity or specificity individually. A calculated $\chi^{2}$ value exceeding the critical value of $\chi_{(2)}^{2}=3.84$ indicated a significant difference. Moreover, the extended McNemar test was used to test the overall significance of the sensitivity and specificity with a fixed error rate; a calculated $\chi^{2}$ value exceeding the critical value of $\chi_{(2)}^{2}=5.99$ indicated a significant difference. The Youden index was applied if one system exhibited significant differences in sensitivity while the other exhibited significant differences in specificity. The highest Youden index denoted the system with the best performance.

The $\kappa$ and weighted $\kappa$ analyses were calculated to determine the interobserver agreement among 3 observers using 2 scales (grades $1-3$ versus grades $4-5$ ) and 3 scales (grades $1-2$ versus grade 3 versus grades $4-5$ ), respectively. $\kappa$ values of $\leq 0.20,0.21-0.40$, $0.41-0.60,0.61-0.80$, and $0.81-1.00$ indicated slight, fair, moderate, substantial, and almost perfect agreement, respectively. ${ }^{15}$ All statistical analyses were 2 -sided, and a $P$ value $<.05$ was a statistically significant difference. The analyses were performed using SPSS software (Version 25.0; IBM, Armonk, New York) and SAS software (Version 9.4; SAS Institute, Cary North Carolina).

\section{RESULTS}

The results for each grade using the current, modified, and plain scan MR imaging systems are shown in Table 2. Metastatic nodes were present in $62.6 \%(154 / 246)$ of patients with stage T1 primary tumors confined to the nasopharynx. All 6 cases with stage T1 NPC detected by nodal assessment alone had abnormal nodes in the upper internal jugular chain, whereas 7/9 patients with benign hyperplasia had abnormal nodes in the retropharyngeal group only. The percentage of patients with NPC in grades 1-2,
3 , and $4-5$ were $0.42 \%$ (1/240), $6.31 \%$ (7/111), and $90.36 \%$ (375/ $415)$, respectively, for the modified grading system, and $0.44 \%$ (1/ 225), $6.96 \%$ (8/115), $87.79 \%$ (374/426), respectively, for the plain scan MR imaging grading system.

The diagnostic performance using the current, modified, and plain scan MR imaging grading systems for grouped grades are presented in Table 3, and the statistical comparisons of the systems are shown in Table 4 . The modified grading system yielded statistically significant better overall performance than the current and the plain scan grading systems. The modified grading system yielded statistically significant better overall performance using the indeterminate grade 3 to indicate benign versus malignant and without nodal assessment versus with nodal assessment.

\section{Observer Results}

The sensitivity, specificity, and accuracy using grades 4 and 5 to indicate malignancy (Table 1) for the plain scan grading system were $96.61 \%, 91.91 \%$, and $94.26 \%$, respectively, for observer 2 , and $95.04 \%, 96.87 \%$, and $95.95 \%$, respectively, for observer 3 ; and for the modified grading system, they were $97.65 \%$, $92.69 \%$, and $95.17 \%$, respectively, for observer 2 and $97.13 \%$, $97.13 \%$, and $97.13 \%$, respectively, for observer 3 . The respective interclass correlation coefficients $(\kappa$ and weighted $\kappa$ ) for the modified systems, which were determined using 2 (grades 1-3 versus grades $4-5$ ) and 3 scales (grades $1-2$ versus grade 3 versus grades $4-5$ ), were 0.87 and 0.81 between observers 1 and 2 and 0.88 and 0.76 between observers 1 and 3 . The respective interclass correlation coefficients for the plain scan systems using 2 and 3 scales were 0.87 and 0.83 between observers 1 and 2 and 0.84 and 0.81 between observers 1 and 3 .

\section{DISCUSSION}

We modified our MR imaging grading system for NPC detection to meet the challenge of using MR imaging to investigate patients with suspected NPC from both the clinical setting (symptomatic) and the screening setting (asymptomatic). We analyzed tumors with a T-stage distribution similar to that expected in a screening population-that is, a high percentage of early-stage primary tumors. Tumors with spread outside the nasopharynx were retained in the analysis to ensure that the grading system reflected the full range of T-stage tumors that may be encountered in both of these settings and to allow us to evaluate the new plain scan 
Table 4: Differences in the diagnostic performances of the current, modified, and plain scan grading systems

\begin{tabular}{|c|c|c|c|c|}
\hline \multirow[b]{2}{*}{ Grading Systems to Indicate NPC } & Sens & Spec & \multicolumn{2}{|l|}{ Combined Sens and Spec } \\
\hline & \multicolumn{2}{|c|}{$\overline{\chi_{(2)}^{2}(\text { Significance, }>3.84)}$} & $\overline{\chi_{(2)}^{2}(\text { Significance, }>5.99)}$ & Youden Index \\
\hline $\begin{array}{l}\text { Current system, primary tumor }(3,4)^{a} \text { vs } \\
\text { Modified system using only primary } \\
\text { tumor }(4,5)^{b}\end{array}$ & $13^{a}$ & $109^{\mathrm{b}}$ & $122^{\mathrm{b}}$ & $a=72.8 \% ; b=88.4 \%$ \\
\hline $\begin{array}{l}\text { Current system, primary tumor }(3,4)^{\mathrm{a}} \text { vs } \\
\text { modified system, primary tumor } \\
(4,5)+\text { node }(5)^{\mathrm{c}}\end{array}$ & $7^{\mathrm{a}}$ & $21.3^{\mathrm{c}}$ & $28.3^{c}$ & $a=72.8 \% ; c=88.1 \%$ \\
\hline $\begin{array}{l}\text { Modified system using only primary } \\
\text { tumor }(4,5)^{d} \text { vs modified system, } \\
\text { primary tumor }(4,5)+\text { node }(5)^{c}\end{array}$ & $6^{\mathrm{c}}$ & $9^{d}$ & $15^{d}$ & $c=88.4 \% ; d=88.1 \%$ \\
\hline $\begin{array}{l}\text { Modified system, primary tumor }(4,5)+ \\
\text { node }(5)^{c} \text { vs plain scan system, } \\
\text { primary tumor }(4,5)+\text { node }(5)^{\mathrm{e}}\end{array}$ & 1 & $5.14^{\mathrm{c}}$ & $6.14^{\mathrm{c}}$ & $c=88.1 \% ; e=85.2 \%$ \\
\hline $\begin{array}{l}\text { Note:-Superscript refers to the grading system wi } \\
\text { Current system, primary tumor }(3,4) \text {. } \\
\text { Modified system using only primary tumor }(4,5) \text {. } \\
\text { Eodified system, primary tumor }(4,5)+\text { node }(5) \text {. } \\
\text { Modified system using only primary tumor }(4,5) \text {. } \\
\text { Plain-scan system, primary tumor }(4,5)+\text { node }(5)\end{array}$ & & & ngeal carcinoma; sens, sensitivit & pecificity. \\
\hline
\end{tabular}

MR imaging grading system that would be advantageous in screening programs.

\section{Current versus Modified Grading Systems for Detection of the Primary Tumor}

The grading system was modified primarily to improve the specificity for primary tumor detection. The current grading system had a low specificity for NPC detection (63.5\%) because of falsepositive results from cases of benign hyperplasia with asymmetry (grade 3). Using grade 4 only in the current grading system (focal mass or extension beyond the nasopharynx) to indicate NPC greatly improved the specificity to $97.9 \%$, but this was at the expense of sensitivity, which decreased from $99.7 \%$ to $87.5 \%$.

The modified grading system yielded a statistically significant better overall performance and better balance between specificity and sensitivity (91.9\% and $96.3 \%$, respectively), producing high positive and negative predictive values $(90.4 \%$ and $97.7 \%$, respectively). This result was achieved mainly by the subdivision of asymmetric diffuse wall thickening into asymmetry without focal expansion, which is less indicative of NPC (new indeterminate grade 3), and into asymmetry with focal expansion, which indicates a higher risk of NPC (new grade 4). Moreover, a focal mass or extension beyond the nasopharynx was elevated to grade 5 , thus expanding the 4 -grade system to 5 grades. The refined criteria also incorporated absent contrast-enhancing septa $^{8}$ and external distortion of contrast-enhancing septa in the adenoid into the new grades 4-5 to indicate suspicious or probable NPC.

\section{Modified Grading System for NPC Detection with and without Metastatic Node Assessment}

The incidence of regional spread to retropharyngeal and upper internal jugular chain nodes covered on the short MR imaging protocol was high for stage T1 tumors confined to the nasopharynx (62.6\%) and is consistent with the reported incidence for early-stage NPC (71.1\%). ${ }^{16}$ The inclusion of nodal assessment increased the sensitivity of the modified grading system by detecting 6 additional cases of NPC that would have been missed using the primary tumor criteria alone. However, nodal assessment also reduced the specificity because of an overlap in the size of enlarged reactive nodes and metastatic nodes. Most interesting, false-positive results for malignancy occurred in the retropharyngeal group despite using the $6-\mathrm{mm}^{13}$ rather than $5-\mathrm{mm}^{17}$ threshold for metastatic node diagnosis. The decrease in specificity outweighed the increase in sensitivity, but the difference in overall performance was small, so we believe that nodal assessment still has a role because it detects primary cancers that would otherwise be missed by MR imaging.

\section{Modified versus Plain Scan Grading Systems for NPC Detection}

As expected, the modified full-protocol grading system, which includes T2- and T1-weighted images before and after contrast enhancement, yielded better overall NPC diagnostic performance than the plain scan grading system. The plain scan grading system, nevertheless, performed well, with high sensitivity and specificity ( $97.7 \%$ and $86.4 \%$, respectively) and high negative and positive predictive values $(97.3 \%$ and $87.8 \%$, respectively). Contrast-enhanced images detected only 1 additional primary cancer and, surprisingly, no additional spread beyond the nasopharynx; this finding is encouraging because it is an important MR imaging indicator of malignancy. Contrast-enhanced images did not detect any additional metastatic nodes. In a screening setting, avoidance of intravenous contrast would enable more rapid, less expensive scans and eliminate the need for intravenous MR imaging contrast agents in healthy patients with false-positive blood tests.

We suggest that for plain scan MR imaging graded 1 or 2 , the risk of NPC is low (0.44\%) and the patient can be reassured. Intravenous contrast is of limited benefit and can be withheld, especially in the absence of abnormal findings on endoscopy. On the other hand, for plain scan MR imaging graded 4 or 5 , the risk of NPC is high (87.80\%) and intravenous contrast is suggested irrespective of the endoscopy findings. For plain scan MR imaging graded 3, the risk of NPC is intermediate (6.96\%) and the role 
of intravenous contrast is unclear, though results suggest that contrast is more beneficial when the MR imaging is graded by a less experienced assessor or to increase the confidence that a symmetric enlarged adenoid is due to benign hyperplasia (striped appearance with alternating septal and lymphoid columns).

\section{Observer Results}

This study aimed to produce a simple MR imaging grading system based on conventional sequences for a quick assessment by radiologists with differing levels of experience. Therefore, we did not include subtle abnormalities of malignancy such as loss of the white line $\operatorname{sign}^{8}$ or functional sequences such as diffusionweighted imaging, ${ }^{18}$ which require quantification and use of thresholds that are not easily implemented across centers. Using our proposed modified and plain scan grading systems yielded substantial agreement between the most and the least experienced observers when assessing grades 1-2 versus grade 3 versus grades 4-5 on contrast MR imaging. All other agreement among the 3 observers was almost perfect. Therefore, we believe that these new MR imaging grading systems can be used by specialists and generalists. Most interesting, the number of false-positive cases increased with experience, which lowered the overall accuracy.

\section{Limitations}

The use of MR imaging in NPC detection is still in its infancy, so our results are from a single-center setting and include cases reported previously. Therefore, to ensure generalizability, the data should be validated at outside institutions, and we hope our proposed systems will be tested and developed by researchers in this field. Moreover, future studies should explore the potential use of only a non-contrast-enhanced MR imaging protocol and the plain scan grading system in screening settings. We envisage future refinements of the MR imaging grading systems, and our proposal regarding the use of intravenous contrast may evolve as advances in the circulating Epstein-Barr virus DNA evaluations reduce the number of healthy subjects referred for further investigation after a false-positive blood test. ${ }^{19}$ Finally, the grading systems are proposed for use only in patients with suspected NPC who have a dedicated nasopharyngeal MR imaging and not for use in a general population.

\section{CONCLUSIONS}

We refined our current MR imaging grading system for NPC detection, with the intent to meet the challenge of applying MR imaging not only in the clinical setting but also in a screening setting involving greater numbers of patients with early-stage primary cancers that must be discriminated from benign hyperplasia. We added focal expansion in diffuse asymmetric wall thickening, absent or external distortion of contrast-enhancing adenoidal septa, and the presence of metastatic nodes as indicators of malignancy. The modified MR imaging grading system improved the overall diagnostic performance of $M R$ imaging when compared with the current MR imaging grading system and produced sensitivity and specificity rates of $96.3 \%$ and $91.9 \%$, respectively. The modified MR imaging grading system was used by specialists and generalists to classify patients into low (grades 1 , 2 ), indeterminate (grade 3), and high-risk (grades 4, 5) categories for NPC. Patients with a low-risk MR imaging grade can be spared an injection of intravenous contrast. We hope the grading system will help disseminate knowledge to other centers and act as the foundation for building a consensus on future guidelines for a Nasopharynx Imaging Reporting and Data System. Finally, the plain scan alone exhibited potential usefulness in future screening programs.

Disclosures: Rossa W.K. Chiu—RELATED: Grant: Research Grants Council of Hong Kong Special Administrative Region Government Theme-Based Research Grant (T12-401/16-W)*; UNRELATED: Consultancy: GRAIL; Grants/Grants Pending: contract research agreement from GRAIL; Patents (Planned, Pending or Issued): both pending and issued patents on cancer diagnostics*; Royalties: from patents on cancer diagnostics*; Stock/Stock Options: GRAIL, Take2 Healthcare; Other: founder of Take2 Healthcare. K.C. Allen Chan-RELATED: Grant: GRAIL, Cirina, Comments: This work is partially supported by GRAIL/Cirina*: Consulting Fee or Honorarium: GRAIL, Comments: I am a consultant to GRAIL; UNRELATED: Board Membership: Take2 Healthcare, DRA, Comments: I am a director of Take2 Healthcare and DRA; Consultancy: GRAIL, Comments: I am a consultant of GRAIL; Grants/Grants Pending: GRAIL/Cirina, Comments: My institution received funding support from GRAIL/Cirina*; Patents (Planned, Pending or Issued): patents on molecular diagnostics; Royalties: GRAIL, Sequenom, Illumine, Take2, Xcelom; Stock/ Stock Options: GRAIL, DRA, Take2 Healthcare, Comments: I hold equities. Wai Kei Jacky Lam—UNRELATED: Patents (Planned, Pending or Issued): enhancement of cancer screening using cell-free viral nucleic acids (pending); Stock/Stock Options: GRAIL. Edwin P. Hui-UNRELATED: Consultancy: Merck Sharp \& Dohme; Payment for Lectures Including Service on Speakers Bureaus: Merck Serono, Merck Sharp \& Dohme. Brigette Ma-UNRELATED: Grants/Grants Pending: Novartis, Boerhinger Ingelheim, Comments: research grant; Payment for Lectures Including Service on Speakers Bureaus: Merck Sharp \& Dohme, Bristol-Myers Squibb.* Anthony ChanUNRELATED: Consultancy: Merck Serono, Merck Sharp \& Dohme, Cullinan Management Inc; Employment. The Chinese University of Hong Kong; Grants/grants pending: Boehringer Ingelheim, Bristol Myers Squibb, Amgen, Pfizer, Eli Lilly, Merck Serono, Merck Sharp \& Dohme*. Yuk Ming Dennis Lo-RELATED: Grant: Kadoorie Charitable Foundation, GRAIL Inc, Li Ka Shing Foundation, Hong Kong Research Grants Council, Comments: Hong Kong Research Grants Council Theme-Based Research Grant (T12-401/16-W)*; Consulting fees or honorarium: GRAIL Inc, Decheng Capital*; Other: Take2 Holdings Limited, GRAIL Inc, Comments: Take2 Holdings Limited - Co-founder, Shareholder, Chairman of the Board, GRAIL Inc. - Scientific Co-founder, shareholder, member of Scientific Advisory Board; UNRELATED: Board membership: DRA Limited, Comments: Chairman of the Board; Patents (Planned, Pending or Issued): Illumina, Sequenom, Xcelom Limited, DRA Limited, Comments: Licensed patents or patent applications in the area of non-invasive prenatal testing; Royalties: Illumina, Sequenom, Xcelom Limited, DRA Limited, Comments: Royalties for licensed patents or patent applications in the area of non-invasive prenatal testing; Stock/stock options: DRA Limited; Other: KingMed Future, Comments: Service agreement in the area of non-invasive prenatal testing. *Money paid to the institution.

\section{REFERENCES}

1. Lee AW, Ng WT, Chan LL, et al. Evolution of treatment for nasopharyngeal cancer: success and setback in the intensity-modulated radiotherapy era. Radiother Oncol 2014;110:377-84 CrossRef Medline

2. Chan KC, Woo JK, King A, et al. Analysis of plasma Epstein-Barr virus DNA to screen for nasopharyngeal cancer. $N$ Engl $J$ Med 2017;377:513-22 CrossRef Medline

3. King AD, Vlantis AC, Bhatia KS, et al. Primary nasopharyngeal carcinoma: diagnostic accuracy of MR imaging versus that of endoscopy and endoscopic biopsy. Radiology 2011;258:531-37 CrossRef Medline

4. King AD, Woo JK, Ai QY, et al. Complementary roles of MRI and endoscopic examination in the early detection of nasopharyngeal carcinoma. Ann Oncol 2019;30:977-82 CrossRef Medline

5. Shayah A, Wickstone L, Kershaw E, et al. The role of cross-sectional imaging in suspected nasopharyngeal carcinoma. Ann R Coll Surg Engl 2019;101:325-27 CrossRef Medline

6. King AD, Vlantis AC, Yuen TW, et al. Detection of nasopharyngeal carcinoma by MR imaging: diagnostic accuracy of MRI compared with endoscopy and endoscopic biopsy based on 
long-term follow-up. AJNR Am J Neuroradiol 2015;36:2380-85 CrossRef Medline

7. King AD, Vlantis AC, Tsang RK, et al. Magnetic resonance imaging for the detection of nasopharyngeal carcinoma. AJNR Am J Neuroradiol 2006;27:1288-91 CrossRef Medline

8. King $\mathrm{AD}$, Wong LY, Law BK, et al. MR imaging criteria for the detection of nasopharyngeal carcinoma: discrimination of earlystage primary tumors from benign hyperplasia. AJNR Am J Neuroradiol 2018;39:515-23 CrossRef Medline

9. Wang ML, Wei XE, Yu MM, et al. Value of contrast-enhanced MRI in the differentiation between nasopharyngeal lymphoid hyperplasia and T1 stage nasopharyngeal carcinoma. Radiol Med 2017;122:743-51 CrossRef Medline

10. Li K, Lin GZ, Shen JC, et al. Time trends of nasopharyngeal carcinoma in urban Guangzhou over a 12-year period (2000-2011): declines in both incidence and mortality. Asian Pac J Cancer Prev 2014;15:9899-903 CrossRef Medline

11. Amin MB, Edge SB, Greene FL, et al, eds. AJCC Cancer Staging Manual. 8th ed. New York: Springer-Verlag; 2017

12. van den Brekel MW, Stel HV, Castelijns JA, et al. Cervical lymph node metastasis: assessment of radiologic criteria. Radiology 1990; 177:379-84 CrossRef Medline
13. Zhang G, Liu L, Wei W, et al. Radiologic criteria of retropharyngeal lymph node metastasis in nasopharyngeal carcinoma treated with radiation therapy. Radiology 2010;255:605-12 CrossRef Medline

14. Hawass N. Comparing the sensitivities and specificities of two diagnostic procedures performed on the same group of patients. Br J Radiol 1997;70:360-66 CrossRef Medline

15. Kundel HL, Polansky M. Measurement of observer agreement. Radiology 2003;228:303-08 CrossRef Medline

16. Guo R, Tang LL, Mao YP, et al. Proposed modifications and incorporation of plasma Epstein-Barr virus DNA improve the TNM staging system for Epstein-Barr virus-related nasopharyngeal carcinoma. Cancer 2019;125:79-89 CrossRef Medline

17. King AD, Ahuja AT, Leung SF, et al. Neck node metastases from nasopharyngeal carcinoma: MR imaging of patterns of disease. Head Neck 2000;22:275-81 CrossRef Medline

18. Ai QY, King AD, Chan JS, et al. Distinguishing early-stage nasopharyngeal carcinoma from benign hyperplasia using intravoxel incoherent motion diffusion-weighted MRI. Eur Radiol 2019;29:5627-34 CrossRef Medline

19. Lam WK, Jiang P, Chan KCA, et al. Sequencing-based counting and size profiling of plasma Epstein-Barr virus DNA enhance population screening of nasopharyngeal carcinoma. Proc Natl Acad Sci U S A 2018;115:E5115-24 CrossRef Medline 